\title{
A New Proof for the Description of Holomorphic Flows on Multiply Connected Domains
}

\author{
F. Jafari, ${ }^{1}$ Z. Słodkowski, ${ }^{2}$ and T. Tonev ${ }^{3}$ \\ ${ }^{1}$ Department of Mathematics, University of Wyoming, Laramie, WY 82071, USA \\ ${ }^{2}$ Department of Mathematics, Statistics and Computer Science, University of Illinois, Chicago, IL 60612, USA \\ ${ }^{3}$ Department of Mathematical Sciences, University of Montana, Missoula, MT 59812, USA
}

Correspondence should be addressed to T. Tonev; tonevtv@mso.umt.edu

Received 14 October 2013; Accepted 6 February 2014; Published 13 March 2014

Academic Editor: S. M. Gusein-Zade

Copyright (C) 2014 F. Jafari et al. This is an open access article distributed under the Creative Commons Attribution License, which permits unrestricted use, distribution, and reproduction in any medium, provided the original work is properly cited.

We provide a new proof for the description of holomorphic and biholomorphic flows on multiply connected domains in the complex plane. In contrast to the original proof of Heins (1941) we do this by the means of operator theory and by utilizing the techniques of universal coverings of the underlying domains of holomorphic flows and their liftings on the corresponding universal coverings.

\section{Introduction}

Holomorphic flows on simply connected domains in the complex plane constitute a rich and well-studied class of maps (cf. [1]). The basic description of holomorphic flows on multiply connected domains is due to Heins [2]. In this paper we provide a new proof of Heins' results and show that, with the notable exception of the punctured disc, every holomorphic flow on a multiply connected domain in the plane is biholomorphic. We do this by the means of operator theory and by utilizing the techniques of universal coverings of the underlying domains of holomorphic flows and their liftings on the universal coverings.

Let $I \subset \mathbb{R}$ be an interval and let $X, Y$ be sets. As customary, a mapping $h: I \times X \rightarrow Y$ can be interpreted as a one-parameter family, $\left\{h_{t}(x)\right\}$, of self-mappings $h_{t}(x)=$ $h(t, x): X \rightarrow Y$, where $t$ runs in $I$ and vice versa.

Definition 1. Let $X$ be a topological space. A family of mappings $\left\{\varphi_{t}\right\}_{[0, \infty]}, \varphi_{t}: X \rightarrow X$ is called a (semigroup) flow on $X$, if $\varphi(t, x) \in C([0, \infty) \times X, X), \varphi_{0}=\mathrm{id}_{X}$, and the composition semigroup rule

$$
\varphi_{s+t}(x)=\varphi_{s}\left(\varphi_{t}(x)\right)=\varphi_{t}\left(\varphi_{s}(x)\right)
$$

holds for every $x \in X, s, t \geq 0$.
A flow is called trivial if all its mappings are equal to the identity on $X$. Flows can be viewed as semigroups of composition operators on $C(X, X)$. The set $X$ is called the underlying domain of the flow. If $\varphi_{t}$ are defined and property (1) holds for every real $t$, then the flow is called a group flow. It is easy to see that if $Y$ is a topological space, $h: X \rightarrow Y$ is a homeomorphism, and the family $\left\{\varphi_{t}\right\}$ is a flow on $X$, then the family $\left\{h \circ \varphi_{t} \circ h^{-1}\right\}$ is a flow on $Y$. A subset $Y \subset X$ is called an invariant space of a flow $\left\{\varphi_{t}\right\}$ on $X$ if all its functions $\varphi_{t}$ map $Y$ into itself. In this case the restrictions $\left.\varphi_{t}\right|_{Y}$ form a flow on $Y$. A singleton invariant space of a flow is called a fixed point of the flow. Namely, $x_{0} \in X$ is a fixed point of a flow $\left\{\varphi_{t}\right\}$ if $\varphi_{t}\left(z_{0}\right)=z_{0}$ for every $t$. By holomorphic (resp., biholomorphic) flow on a domain $G \subset \mathbb{C}$ in this paper we will understand, as usual, a flow $\left\{\varphi_{t}\right\}$ on $G$ such that all $\varphi_{t}$ are holomorphic (resp., biholomorphic) functions from $G$ into $G$.

\section{Holomorphic Flows on Multiply Connected Domains in $\mathbb{C}$}

Holomorphic flows on simply connected domains in the complex plane $\mathbb{C}$ are well studied. As shown in [1], any holomorphic flow on $\mathbb{C}$ is biholomorphic. 
Theorem 2 (see [1]). If $\varphi=\{\varphi(t, \cdot)\}$ is a holomorphic flow on $\mathbb{C}$, then one of the following holds:

(i) $\varphi$ does not have fixed points in $\mathbb{C}$ and $\varphi(t, z)=z+K t$ for some $K \in \mathbb{C}$.

(ii) $\varphi$ has one fixed point $z=z_{0}$ in $\mathbb{C}$ and $\varphi(t, z)=e^{\alpha t} z+$ $z_{0}\left(1-e^{\alpha t}\right)$.

Recall that any 2-connected domains $G$ in $\mathbb{C}$ are biholomorphic to one of the three canonical 2-connected domains: $\mathbb{C} \backslash\{0\}, \mathbb{D} \backslash\{0\}$, and an annulus.

Let $\left\{\varphi_{t}\right\}$ be a holomorphic flow on $\mathbb{C} \backslash\{0\}$ or $\mathbb{D} \backslash\{0\}$. Since each of the functions $\varphi_{t}$ is homotopic to the $\varphi_{0}=\left.\mathrm{id}\right|_{G}$, it follows that every $\varphi_{t}$ is bounded near $\{0\}$ and, therefore, its singularity at $\{0\}$ can be removed by the Riemann's singularity theorem. Thus any $\varphi_{t}$ can be extended at $\{0\}$ as $\varphi_{t}(0)=0$, and together the whole flow $\left\{\varphi_{t}\right\}_{[0, \infty)}$ can be extended to a holomorphic flow; say $\left\{\widetilde{\varphi}_{t}\right\}_{[0, \infty)}$, on $\mathbb{C}$ or on $\mathbb{D}$ correspondingly, which fixes 0 .

As shown in [1] any biholomorphic flow $\left\{\varphi_{t}\right\}$ on the unit disc $\mathbb{D}$ consists of Möbius transformations, and if 0 is a fixed point of this flow, then $\varphi_{t}(z)=e^{i \theta t}$ for some $\theta \in \mathbb{R}$. However, in $\mathbb{D} \backslash\{0\}$ there exist holomorphic flows that are not biholomorphic. Namely, as shown in [1], any holomorphic flow on $\mathbb{C}$ with a fixed point, restricted to an invariant domain of the flow containing the fixed point, generates a holomorphic flow on $\mathbb{D} \backslash\{0\}$, which is not necessarily biholomorphic. Here are two examples.

Example 3. The flow $\varphi(t, z)=e^{\alpha t} z$ with $\alpha \in \mathbb{C}, \operatorname{Re} \alpha \leq 0$, is a holomorphic flow on $\mathbb{D} \backslash\{0\}$. It is biholomorphic if and only if $\operatorname{Re} \alpha=0$.

Example 4. $\varphi(t, z)=\beta e^{-t} z /\left(\left(e^{-t}-1\right) z+\beta\right),|\beta|=1$, is a holomorphic flow on $\mathbb{D} \backslash\{0\}$, which is not biholomorphic.

As mentioned above, the holomorphic flows on $\mathbb{C} \backslash\{0\}$ are restrictions on $\mathbb{C} \backslash\{0\}$ of holomorphic flows on $\mathbb{C}$ that fix 0 . Theorem 2 implies that these are only the flows of type $\varphi(t, z)=e^{\alpha t} z, \alpha \in \mathbb{C} \backslash\{0\}$, all of which are biholomorphic.

The next theorem describes holomorphic flows on the other multiply connected domains in $\mathbb{C}$.

Theorem 5 (see [2]). (a) Every holomorphic (and biholomorphic) flow $\varphi$ on an annulus $G$ is of type $\varphi(t, z)=e^{i \theta t} z$ for some real $\theta$. (b) Every holomorphic (and biholomorphic) flow $\varphi$ on a $k$-connected domain in $\mathbb{C}, k>2$, is trivial.

In contrast to Heins' original approach we prove this theorem by utilizing the techniques of universal coverings and of liftings of holomorphic flows on the universal coverings of their underlying domains in $\mathbb{C}$ in an operator theoretic setting. First we provide several preliminary results that are interesting on their own.

Lemma 6. Consider the strip $S=\{z \in \mathbb{C}: 0<\operatorname{Re} z<1\}$. Let $T_{0}>0$ be a fixed positive number, and let $\tau_{0}: S \rightarrow S$ be the mapping $\tau_{0}(z)=z+i T_{0}$. Any holomorphic map $f$ of $S$ into itself that commutes with $\tau_{0}$ has the form $f(z)=z+i T$ for some real $T$.
Proof. Let $f(x, y)=u(x, y)+i v(x, y)$. Denote $w(x, y)=$ $\int_{0}^{T_{0}} u(x, y+\eta) d \eta$. Clearly, $w: S \rightarrow \mathbb{R}$ is a harmonic function. The commuting relation $f \circ \tau_{0}=\tau_{0} \circ f$, that is, $f\left(z+i T_{0}\right)=$ $f(z)+i T_{0}$, implies

$$
\begin{gathered}
u\left(x, y+T_{0}\right)=u(x, y), \\
v\left(x, y+T_{0}\right)=v(x, y)+T_{0} .
\end{gathered}
$$

Thus, for every $x$ the function $u(x, \cdot)$ is periodic in $y$, with period $T_{0}$. Hence, the integral over any interval with length $T_{0}$ is constant; that is, $w(x, y)=w_{0}(x)$. Now $w_{0}(x)$ is a harmonic function, and so it is linear; say, $w_{0}(x)=w(x, y)=A+B x$. Observe that

$$
\begin{aligned}
T_{0} & =v\left(x_{0}, y_{0}+T_{0}\right)-v\left(x_{0}, y_{0}\right)=\int_{y_{0}}^{y_{0}+T_{0}} \frac{\partial v}{\partial y}\left(x_{0}, y\right) d y \\
& =\int_{y_{0}}^{y_{0}+T_{0}} \frac{\partial u}{\partial x}\left(x_{0}, y\right) d y=\left(\frac{\partial}{\partial x} \int_{y_{0}}^{y_{0}+T_{0}} u(x, y) d y\right)\left(x_{0}\right) \\
& =\frac{\partial w}{\partial x}\left(x_{0}, y_{0}\right)=\frac{\partial(A+B x)}{\partial x}\left(x_{0}, y_{0}\right)=B
\end{aligned}
$$

Therefore, $B=T_{0}$ and $w_{0}(x)=A+T_{0} x$; that is, $\left(1 / T_{0}\right) \int_{y_{0}}^{y_{0}+T_{0}} u(x, y) d y=A / T_{0}+x$ for any $0<x<1$. Note that $0 \leq A / T_{0}+x \leq 1$ for any $0<x<1$, since $0 \leq u(x, y) \leq 1$. Hence, $A=0$; that is, $\left(1 / T_{0}\right) \int_{y_{0}}^{y_{0}+T_{0}} u(x, y) d y=x$ for any $0<x<1$. Denote $u^{*}(1, y)=\lim _{x \rightarrow 1^{-}} u(x, y)$, where the limit exists $d y$-almost everywhere on $\mathbb{R}$. Since $1-u^{*}(1, y) \geq$ 0 , we have $\left(1 / T_{0}\right) \int_{y_{0}}^{y_{0}+T_{0}}\left(1-u^{*}(1, y)\right) d y=0$ for every $y_{0}$. Therefore, $1-u^{*}(1, y)=0$; that is, $u^{*}(1, y)=1 d y$-almost everywhere. Similarly, for $u^{*}(0, y)=\lim _{x \rightarrow 0^{+}} u(x, y)$ we have $u^{*}(0, y) \geq 0$, and $\left(1 / T_{0}\right) \int_{y_{0}}^{y_{0}+T_{0}} u^{*}(0, y) d y=0$ for every $y_{0}$; thus $u^{*}(0, y)=0 d y$-almost everywhere. Since $u(x, y)$ is a bounded harmonic function, we obtain that $u(x, y)=x$, and therefore, $v(x, y)=y+T$; that is, $f(z)=z+i T$ for some real number $T$.

Recall that a Möbius transformation $\sigma: \mathbb{D} \rightarrow \mathbb{D}$ is hyperbolic if it has exactly two distinct fixed points in $b \mathbb{D}$, the topological boundary of $\mathbb{D}$; that is, $\sigma(p)=p, \sigma(q)=q$ for some $p, q \in b \mathbb{D}, p \neq q$. Denote by $H_{p, q}$ the set of all hyperbolic transformations $\sigma: \overline{\mathbb{D}} \rightarrow \overline{\mathbb{D}}$ with common fixed points $p, q \in b \mathbb{D}$, together with the identity map.

Lemma 7. Let $\sigma: \mathbb{D} \rightarrow \mathbb{D}$ belong to $H_{p, q}$. If $\Phi: \mathbb{D} \rightarrow \mathbb{D}$ is a holomorphic map commuting with $\sigma$, then $\Phi \in H_{p, q}$. The set of all such maps, namely, $H_{p, q}$, forms a one-parameter group.

Proof. Let $p$ and $q \in b \mathbb{D}$ be the fixed points of $\sigma$. Denote by $S$ the strip $S=\{z \in \mathbb{C}: 0<\operatorname{Re} z<1\}$. Let $H: \overline{\mathbb{D}} \rightarrow \bar{S}$ denote the homeomorphic extension of the Riemann mapping of $\mathbb{D}$ onto $S$. $H$ maps $\overline{\mathbb{D}}$ onto the compactification $\bar{S}=S \cup(\{0\} \times$ $i \mathbb{R}) \cup(\{1\} \times i \mathbb{R}) \cup\{-i \infty\} \cup\{\mathrm{i} \infty\}$. We may choose $H$ so that $H(p)=-i \infty$, and $H(q)=i \infty$. Transfer the mappings $\sigma$ and 
$\Phi$ to $S$, by setting $g=H \circ \sigma \circ H^{-1}$ and $f=H \circ \Phi \circ H^{-1}$. We have that $g(-\infty)=-\infty, g(i \infty)=i \infty,\left.g\right|_{S}$ is conformal, and $f$ commutes with $g$. Hence $g$ is a shift in $S$; that is, there is a $T_{0} \in \mathbb{R}$ such that $g(z)=z+i T_{0}$. Lemma 6 implies that $f(z)=z+i T$ for some $T \in \mathbb{R}$. Obviously, the set of all such $f$ is a one-parameter group $\left\{f_{T}: T \in \mathbb{R}\right\}$ of shifts on $S$ with $f_{T}: \bar{S} \rightarrow \bar{S}: \varphi_{T}(z)=z+i T$ with two common distinct fixed points $-i \infty$ and $+i \infty$. Finally, $F_{T}=H^{-1} \circ f_{T} \circ H, T \in$ $\mathbb{R}$ (including $F$ ), are all conformal transformations of $\mathbb{D}$, that is, Möbius transformations with two common distinct points $p, q \in b \mathbb{D}$. Clearly, $\left\{F_{T}\right\}$ is a one-parameter group on $\mathbb{D}$.

Recall some facts about universal covering spaces of domains in $\mathbb{C}$. Let $G$ be a domain in $\mathbb{C}$, or, an open Riemann surface. Let $U \subset \mathbb{C}$ be the (simply connected) universal covering space of $G$, the covering mapping (the projection) $\pi: U \rightarrow G$ of which is a local homeomorphism. Note that since $U$ is simply connected, then $U$ is homeomorphic either to $\mathbb{D}$ or to $\mathbb{C}$. It is clear that under the complex structure on $U$ pulled back by $\pi$ from $G, \pi$ is a holomorphic map. There is a group $\Gamma=\{\gamma\}$ of Möbius transformations $\gamma(\xi)$ of $U$ onto itself with the following properties:

(i) $\Gamma$ acts discretely on $U$;

(ii) $\Gamma\left(\xi_{0}\right)=\left\{\gamma\left(\xi_{0}\right): \gamma \in \Gamma\right\}=\pi^{-1}\left(\pi\left(\xi_{0}\right)\right)$ for every fixed $\xi_{0} \in U$;

(iii) $\pi \circ \gamma=\pi$ for every $\gamma \in \Gamma$.

Recall the standard construction and properties of the universal covering $U$ of $G$ (cf. [3]) that we will need in the sequel. If $X$ and $Y$ are topological spaces and $I$ is an interval in $\mathbb{R}$, then a jointly continuous mapping $F: I \times X \rightarrow Y$ is called a homotopy on $X$. As mentioned above, the homotopy $F$ will be denoted also by $\left\{F_{t}\right\}_{I}, F_{t}: X \rightarrow Y$. If $X, Y$ are topological spaces and $f_{1}, f_{2} \in C(X, Y)$, then a homotopy connecting $f_{1}$ and $f_{2}$ is any continuous function $F(t, x):[0,1] \times X \rightarrow Y$ such that $F(0, x) \equiv f_{0}(x)$, and $F(1, x) \equiv f_{1}(x)$. Two mappings $f_{1}, f_{2} \in C(X, Y)$ are called homotopic to each other if there exists a homotopy that connects them.

Consider the pointed space $\left\{G, z_{0}\right\}$. The covering space $U$ of $G$ is defined as the set of homotopy classes (with both ends fixed) $\xi=[\tau]$ of the family of all paths $\tau:[0,1] \rightarrow G$ with initial point $\tau(0)=z_{0}$, equipped with the standard topology. The covering mapping $\pi: U \rightarrow G$ is defined by $\pi(\xi)=\tau(1)$, where $\xi=[\tau]$. The group $\Gamma=\{\gamma\}$ is defined as the family of homotopy classes $\gamma=\left[\tau_{1}\right]$ of closed loops $\tau_{1}$ with $\tau_{1}(1)=$ $\tau_{1}(0)=z_{0}$. Such $\gamma$ acts on any $\xi=[\tau] \in U$ by $\gamma(\xi)=\left[\tau_{1}\right] \star$ $[\tau]=\left[\tau_{1} \tau\right]$, where $\tau_{1} \tau$ is the end-to-end path initiating at $z_{0}=\tau(0)$, terminating at $\tau_{1}(1)$, with $\tau(1)=\tau_{1}(0)$ (see, e.g., [3, Ch. 1, Sec. 7]) for definition of the path groupoid.

In the case of $X=[0,1]$ the next lemma is proven in (cf. [3, Ch. 2, Sec. 2, Theorems 2 and 3]). The proof of the general case goes along similar lines.

Lemma 8 (uniqueness of homotopy lifting). Let $X$ be a connected space, $\widetilde{h}: X \rightarrow U$ a fixed continuous mapping, and $\left\{h_{t}\right\}_{[0, \infty)}, h_{t}: X \rightarrow G$ a homotopy on $X$ such that $h_{0}=\pi \circ \tilde{h}$.
Then there is a unique homotopy $\left\{\tilde{h}_{t}\right\}_{[0, \infty)}$ with $\tilde{h}_{t}: X \rightarrow U$ such that
(i) $\widetilde{h}_{0}=\widetilde{h}$;
(ii) $\pi \circ \widetilde{h}_{t}=h_{t}$ for every $t \geq 0$.

Clearly, every function $\varphi_{t}$ of a flow $\left\{\varphi_{t}\right\}$ on $X$ is homotopic to id| $\left.\right|_{X}=\varphi_{0}$.

Corollary 9. Let $G$ be a proper subset of $\mathbb{C}$ with universal covering $U$, and let $\Gamma$ be the corresponding group of Möbius transformations. Assume that $\left\{\varphi_{t}\right\}_{[0, \infty)}$ is a homotopy on $G$ with $\varphi_{0}=i d_{G}$. Then there is a unique homotopy $\left\{\Phi_{t}\right\}_{[0, \infty)}$, $\Phi_{t}: U \rightarrow U$, such that

(1) $\Phi_{0}=i d_{U}$

(2) $\pi \circ \Phi_{t}=\varphi_{t} \circ \pi$ for all $t \geq 0$;

(3) $\gamma \circ \Phi_{t}=\Phi_{t} \circ \gamma$ for every $\gamma \in \Gamma$ and all $t \geq 0$.

Proof. We apply Lemma 8 with $X=U, \widetilde{h}=\mathrm{id}_{U}$ and the homotopy $\left\{h_{t}\right\}_{[0, \infty)}=\left\{\varphi_{t} \circ \pi\right\}_{[0, \infty)}$ with $\varphi_{t} \circ \pi: U \rightarrow G$. Clearly, $\pi \circ \mathrm{id}_{U}=\varphi_{0} \circ \pi=\pi$. By Lemma 8 there is a homotopy $\left\{\tilde{h}_{t}\right\}_{[0, \infty)}$ with $\widetilde{h}_{t}: U \rightarrow U$, such that $\pi \circ \tilde{h}_{t}=h_{t}=\varphi_{t} \circ \pi$, and $\tilde{h}_{0}=\tilde{h}=\mathrm{id}_{U}$. If we denote $\tilde{h}_{t}$ by $\Phi_{t}$, then, clearly, conditions (1) and (2) are satisfied. The homotopy $\left\{\Phi_{t}\right\}_{[0, \infty)}$ is uniquely defined, for two homotopies $\left\{\Phi_{t}\right\},\left\{\Phi_{t}^{*}\right\}$ on $U$ with $\Phi_{0}=\Phi_{0}^{*}=\operatorname{id}_{U}$ and $\pi \circ \Phi_{t}=\pi \circ \Phi_{t}^{*}=\varphi_{t} \circ \pi$ being two liftings of the homotopy $h_{t}=\varphi_{t} \circ \pi, t \geq 0$, in contradiction with Lemma 8.

To prove condition (3), fix a $\gamma \in \Gamma$ and define $\Phi_{t}^{*}=\gamma$ 。 $\Phi_{t} \circ \gamma^{-1}$. Clearly, $\Phi_{0}^{*}=\gamma \circ \mathrm{id}_{U} \circ \gamma^{-1}=\mathrm{id}_{U}$, and, by property (iii) of covering mappings, $\pi \circ \Phi_{t}^{*}=\pi \circ\left(\gamma \circ \Phi_{t} \circ \gamma^{-1}\right)=$ $\pi \circ \Phi_{t} \circ \gamma^{-1}=\varphi_{t} \circ\left(\pi \circ \gamma^{-1}\right)=\varphi_{t} \circ \pi$. Thus, $\left\{\Phi_{t}\right\}_{[0, \infty)}$ and $\left\{\Phi_{t}^{*}\right\}_{[0, \infty)}$ are two homotopies on $U$ that satisfy conditions (1) and (2). By the uniqueness of homotopy liftings (Lemma 8) we obtain $\Phi_{t}=\Phi_{t}^{*}=\gamma \circ \Phi_{t} \circ \gamma^{-1}$. Hence, $\Phi_{t} \circ \gamma=\gamma \circ \Phi_{t}$ for any $t \geq 0$.

Corollary 10. Under the notations and assumptions of Corollary 9, if the homotopy $\left\{\varphi_{t}\right\}_{[0, \infty)}$ is a flow on a set $G \subset \mathbb{C}$, then the lifted homotopy $\left\{\Phi_{t}\right\}_{[0, \infty)}$ is a flow on $U$.

Proof. Fix a $t_{0}>0$ and define a new homotopy $\left\{\Phi_{t}^{*}\right\}_{[0, \infty)}$, $\Phi_{t}^{*}: U \rightarrow U$, by

$$
\Phi_{t}^{*}= \begin{cases}\Phi_{t} & \text { when } 0 \leq t \leq t_{0} \\ \Phi_{t-t_{0}} \circ \Phi_{t_{0}} & \text { when } t \geq t_{0}\end{cases}
$$

where $\left\{\Phi_{t}\right\}$ is the lifted by Corollary 9 homotopy. By the continuity argument, $\Phi_{t}^{*}$ is well defined and satisfies conditions (1) and (2) from Corollary 9. Indeed, $\Phi_{0}^{*}=\Phi_{0}=\mathrm{id}_{U}$, and $\pi \circ \Phi_{t}^{*}=\pi \circ \Phi_{t}=\varphi_{t} \circ \pi$ for all $0 \leq t \leq t_{0}$. The semigroup property (1) of $\varphi_{t}$ implies that for any $t>t_{0}$ we have

$$
\begin{aligned}
\pi \circ \Phi_{t}^{*} & =\pi \circ \Phi_{t-t_{0}} \circ \Phi_{t_{0}}=\varphi_{t-t_{0}} \circ\left(\pi \circ \Phi_{t_{0}}\right) \\
& =\left(\varphi_{t-t_{0}} \circ \varphi_{t_{0}}\right) \circ \pi=\varphi_{t} \circ \pi .
\end{aligned}
$$


By the uniqueness part of Corollary 9 it follows that $\Phi_{t}^{*}=\Phi_{t}$. Therefore, for any $t>t_{0}$ we have $\Phi_{t-t_{0}} \circ \Phi_{t_{0}}=\Phi_{t}$. Hence $\left\{\varphi_{t}\right\}_{[0, \infty)}$ is a (semigroup) flow on $U$.

In the next corollary we summarize the above results.

Corollary 11. Let $G$ be a k-connected domain in $\mathbb{C}, k>$ 2 , with universal covering $U$ and corresponding group of Möbius transformations $\Gamma$. If $G$ admits other than the identity holomorphic self-map $\varphi: G \rightarrow G$, then either

(i) $\Gamma$ does not contain any hyperbolic Möbius transformations, or

(ii) $\Gamma$ is commutative, in fact an infinite cyclic group, and $G$ is homeomorphic to an annulus.

Proof. Suppose that the group $\Gamma$ contains a hyperbolic transformation $\sigma$ with fixed points $p \neq q$; that is $\sigma \in H_{p, q}, \sigma \neq \mathrm{id}_{U}$. Let $\varphi: G \rightarrow G, \varphi \neq \mathrm{id}_{G}$ be a holomorphic function on $G$. Since the lifting $\Phi: U \rightarrow U$ of $\varphi$ commutes with $\Gamma$, it commutes also with $\sigma$. Lemma 7 implies that $\Phi \in H_{p, q} \backslash$ $\left\{\mathrm{id}_{U}\right\}$. Again by Lemma 7, with $\sigma=\Phi$, we see that every holomorphic self-map of $U$ commuting with $\sigma$ belongs to $H_{p, q}$. Therefore, $\gamma \in H_{p, q}$ for every $\gamma \in \Gamma$, thus $\Gamma \subset H_{p, q}$. Since $H_{p, q}$ is a commutative one-parameter group and $\Gamma$ is discrete, we conclude that $\Gamma$ is a cyclic group. Therefore, $G \cong$ $U / \Gamma \cong \mathbb{D} / \Gamma$ is homeomorphic to an annulus and not to the punctured disc.

Let $\varphi$ be a holomorphic function on a $k$-connected domain that is homotopic to the identity, $\left\{\varphi_{t}\right\}_{[0,1]}$ be a homotopy on $G$ connecting $\varphi=\varphi_{1}$ with $\operatorname{id}_{G}=\varphi_{0}$, and $\left\{\Phi_{t}\right\}_{[0,1]}$ be the unique, by Corollary 9 , lifting of the family $\left\{\varphi_{t}\right\}_{[0,1]}$ on the universal covering $U \cong \mathbb{D}$ of $G$ with $\Phi_{0}=\mathrm{id}_{U}$.

Lemma 12. If $G$ is a non-simply-connected domain in $\mathbb{C}$ and $\varphi: G \rightarrow G, \varphi \neq i d_{G}$ is a holomorphic function on a $k$ connected domain that is homotopic to the identity, then both $\varphi$ and its lifting $\Phi$ on the covering $U$ of $G$ are fixed-point free.

Proof. Since $G$ is multiply connected, then its universal covering $U$ is homeomorphic to the unit disc $\mathbb{D}$. Suppose that $z_{0}$ is a fixed point for $\varphi$; that is, $\varphi\left(z_{0}\right)=z_{0}$. Since $(\pi \circ \Phi)(\xi)=$ $(\varphi \circ \pi)(\xi)$ on $U$, we have that $\Phi(\xi) \subset \pi^{-1}((\varphi \circ \pi)(\xi))$ for any $\xi \in U$. Therefore, $\Phi\left(\pi^{-1}\left(z_{0}\right)\right) \subset \pi^{-1}\left(\varphi\left(z_{0}\right)\right)=\pi^{-1}\left(z_{0}\right)$. We claim that $\left.\Phi\right|_{\pi^{-1}\left(z_{0}\right)}=\mathrm{id}_{\pi^{-1}\left(z_{0}\right)}$. To proof this we show first that there is a $\xi_{0} \in \pi^{-1}\left(z_{0}\right)$ such that $\Phi\left(\xi_{0}\right)=\xi_{0}$.

First we describe in detail the lifting $\left\{\widetilde{\Phi}_{t}\right\}$ of the homotopy $\left\{\varphi_{t}\right\}$ connecting $\varphi=\varphi_{1}$ and $\varphi_{0}=\mathrm{id}_{G}$, so that $\Phi_{0}=\mathrm{id}_{U}$. For any given path $\tau:[0,1] \rightarrow G$ in $G$ with initial point $\tau(0)=z_{0}$ and $\xi=[\tau]$ we define $\widetilde{\Phi}_{t}$ as $\widetilde{\Phi}_{t}(\xi)=\left[\varphi_{t} \circ \tau\right]$, $\varphi_{t} \circ \tau:[0,1] \rightarrow G,\left(\varphi_{t} \circ \tau\right)(s)=\varphi_{t}(\tau(s))$. Clearly, the mapping $\widetilde{\Phi}_{t}$ satisfies properties (1), (2) and (3) of Corollary 9. In particular, $\widetilde{\Phi}_{0}(\xi)=\xi=\Phi_{0}(\xi)$ on $U$. The uniqueness of the homotopy lifting implies that $\Phi_{t}=\widetilde{\Phi}_{t}$, and, in particular, $\Phi=\widetilde{\Phi}_{1}$.

Consider now the constant loop $\tau_{0}:[0,1] \rightarrow G$ : $\tau(t)=z_{0}$, and let $\xi_{0}=\left[\tau_{0}\right]$. Вy $\varphi \circ \tau_{0}=\tau_{0}$, we have that $\Phi\left(\xi_{0}\right)=\widetilde{\Phi}_{1}\left(\xi_{0}\right)=\left[\varphi \circ \tau_{0}\right]=\left[\tau_{0}\right]=\xi_{0}$; that is, $\xi_{0}$ is a fixed point of $\Phi$. Since $\pi^{-1}\left(z_{0}\right)=\Gamma\left(\xi_{0}\right)=\left\{\gamma\left(\xi_{0}\right): \gamma \in \Gamma\right\}$ and since $\Phi$ commutes with all $\gamma \in \Gamma$, we see that $\Phi\left(\gamma\left(\xi_{0}\right)\right)=$ $\gamma\left(\Phi\left(\xi_{0}\right)\right)=\gamma\left(\xi_{0}\right)$. Hence, every point of type $\gamma\left(\xi_{0}\right) \in U$ is a fixed point of $\Phi$. Since $U$ is a disc, the holomorphic mapping $\Phi: U \rightarrow U$ with more than one fixed point must be the identity. Consequently, $\varphi=\pi \circ \Phi=\mathrm{id}_{G}$, in contradiction with the hypotheses.

Proposition 13. The only holomorphic mapping on a $k$ connected domain in $\mathbb{C}, k>2$, that is homotopic to the identity is the identity itself.

Proof. Let $\varphi \neq\left.\mathrm{id}\right|_{G}$ be a map as in Theorem 5, and let $\Phi$ be the lifting of $\varphi$ on the universal covering $U$ of $G$ that commutes with the group $\Gamma$, that is, such that $\Phi \circ \gamma=\gamma \circ \Phi$ for any $\gamma \in \Gamma$. By Lemma 12, $\Phi$ has no fixed points in $U \cong \mathbb{D}$, since $G$ is not simply connected. By the Denjoy-Wolff theorem, there is an $\alpha \in b U$, such that for every $\xi \in U$ we have $\lim _{n \rightarrow \infty} \Phi^{n}(\xi)=\alpha$, where $\Phi^{n}$ is the $n$th composition of $\Phi$ with itself. We have also that $\lim _{n \rightarrow \infty} \Phi^{n}(\gamma(\xi))=\alpha$ for every $\gamma \in \Gamma$. Since $\Phi^{n}(\gamma(\xi))=\gamma \circ \Phi^{n}(\xi)$, it follows that $\alpha=$ $\lim _{n \rightarrow \infty} \Phi^{n}(\gamma(\xi))=\gamma \lim _{n \rightarrow \infty} \Phi^{n}(\xi)=\gamma \alpha$. Therefore, $\gamma \alpha=\alpha$ for every $\gamma \in \Gamma$. Thus all Möbius transformations in $\Gamma$ have a common fixed point $\alpha$. If we map $U \cong \mathbb{D}$ onto the upper halfplane $H=\{z: \operatorname{Im} z>0\}$ by a Möbius transformation, so that $\alpha$ is mapped at the point at $\infty$ and transfer $\Gamma$ on $H$, then $\Gamma$ consists of Möbius mappings preserving the half-plane $H$ with fixed points at $\infty$, that is, of type $\zeta \mapsto A \zeta+B$ for some real $A, B, A \neq 0$.

According to Corollary 11, if $\Gamma$ contains a hyperbolic mapping $\gamma$, then it is homeomorphic to an annulus. Hence $G$ is 2-connected, in contradiction with the hypotheses. If $G$ is not homeomorphic to an annulus, then all elements of $\Gamma$ must be parabolic, elliptic, or, the identity. No transformation of type $\gamma(\zeta)=A \zeta+B$ with $A, B$ real, can be elliptic, and, therefore, $\gamma$ is parabolic; hence $A=1$. Consequently, the members of $\Gamma$ are translations $\zeta \mapsto \zeta+B, B \in \mathbb{R}$. Since $\Gamma$ is discrete, it must be an infinite cyclic group of the form $\Gamma=\left\{\gamma_{0}^{n}: n \in \mathbb{Z}\right\}$, where $\gamma_{0}(\zeta)=z+B_{0}$ with some $B_{0}>0$. Hence, the domain $G \cong H / \Gamma \cong U / \Gamma$ is homeomorphic to the punctured disc, which implies that $G$ is 2-connected, contrary to its hypothesis. This completes the proof.

Proof of Theorem 5. (a) If $G$ is an annulus in $\mathbb{C}$, then its universal covering is the $\operatorname{strip} S=\{z: 0<\operatorname{Im} z<1\}$. Let $\left\{\varphi_{t}\right\}$ be a holomorphic flow on $G$, and $\left\{\Phi_{t}\right\}$ is its lifted flow on $S$. Each $\Phi_{t}$ commutes with the group $\Gamma$ of corresponding Möbius transformations, which consists of translations $z+i T$, $T \in \mathbb{R}$. Let $\tau_{0}=z+i T_{0} \in \Gamma$ with $T_{0}>0$. Since $\Phi_{t} \circ \tau_{0}=\tau_{0} \circ \Phi_{t}$, Lemma 6 implies that $\Phi_{t}(z)=z+i T_{t}, T_{t} \in \mathbb{R}$. Therefore the semigroup flow $\left\{\Phi_{t}\right\}$ consists of translations $\{z+i T, T \in \mathbb{R}\}$, whose projection $\varphi_{t}$ on the annulus $G$ are rotations. Hence for every $t \geq 0, \varphi_{t}(z)=e^{i \theta_{t}} z$ for some real $\theta_{t}$. The semigroup property implies that there is a real $\theta$ such that $\varphi_{t}(z)=e^{i \theta t} z$.

(b) Let $\varphi=\left\{\varphi_{t}\right\}$ be a holomorphic flow on a $k$-connected domain in $\mathbb{C}, k>2$. Since the mappings $\varphi_{t}$ are holomorphic functions on $G$, then their liftings $\Phi_{t}$ on $U$, the universal covering of $G$ are holomorphic mappings on $U$, since the projection $\pi$ is holomorphic. The proposition from the above implies that the flow $\varphi$ is trivial. 


\section{Conflict of Interests}

The authors declare that there is no conflict of interests regarding the publication of this paper.

\section{Acknowledgment}

The research of T. Tonev was partially supported by Grant no. 209762 from the Simons Foundation.

\section{References}

[1] F. Jafari, T. Tonev, E. Toneva, and K. Yale, "Holomorphic flows, cocycles, and coboundaries," Michigan Mathematical Journal, vol. 44, no. 2, pp. 239-253, 1997.

[2] M. H. Heins, "On the iteration of functions which are analytic and single-valued in a given multiply-connected region," American Journal of Mathematics, vol. 63, pp. 461-480, 1941.

[3] E. H. Spanier, Algebraic Topology, Springer, New York, NY, USA, 1982. 


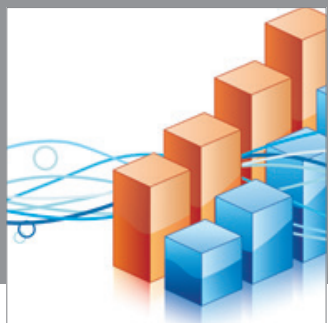

Advances in

Operations Research

mansans

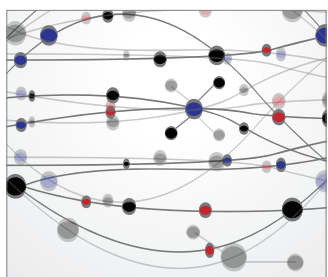

The Scientific World Journal
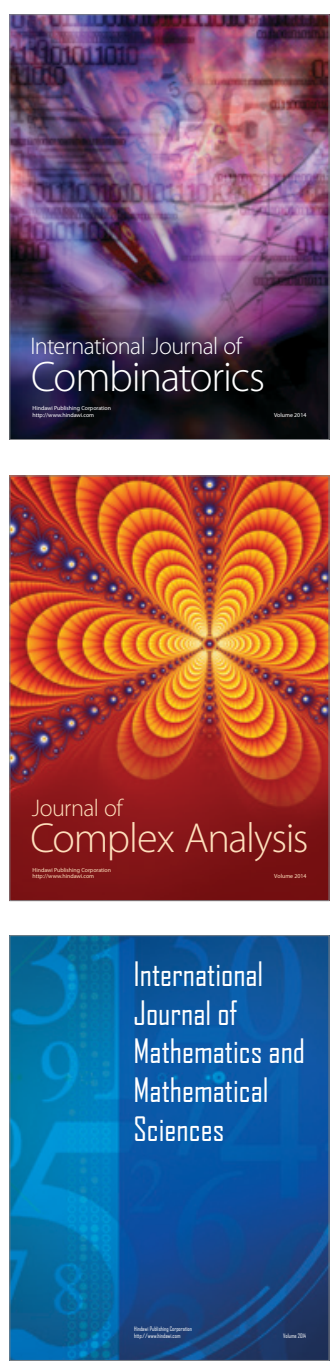
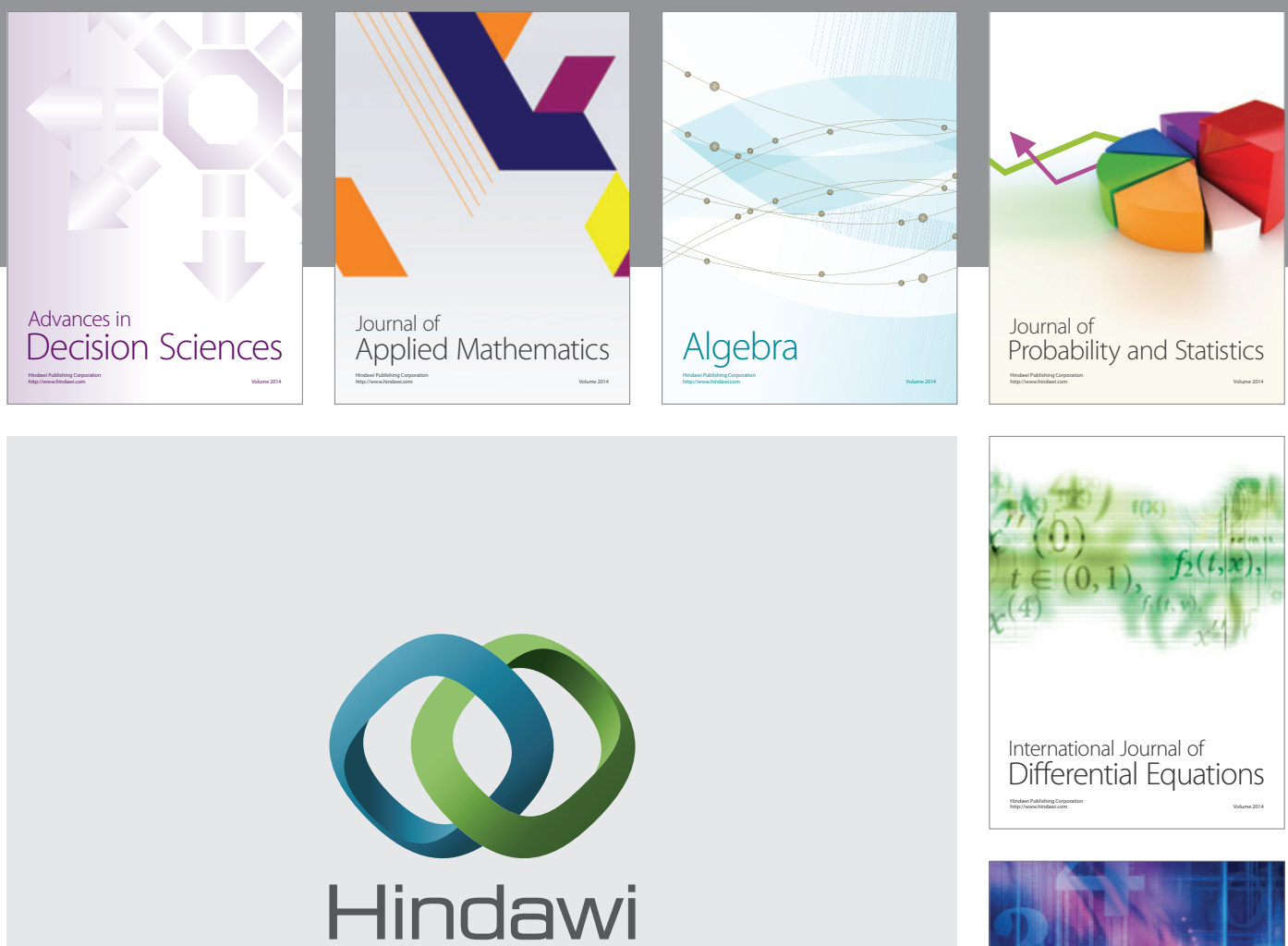

Submit your manuscripts at http://www.hindawi.com
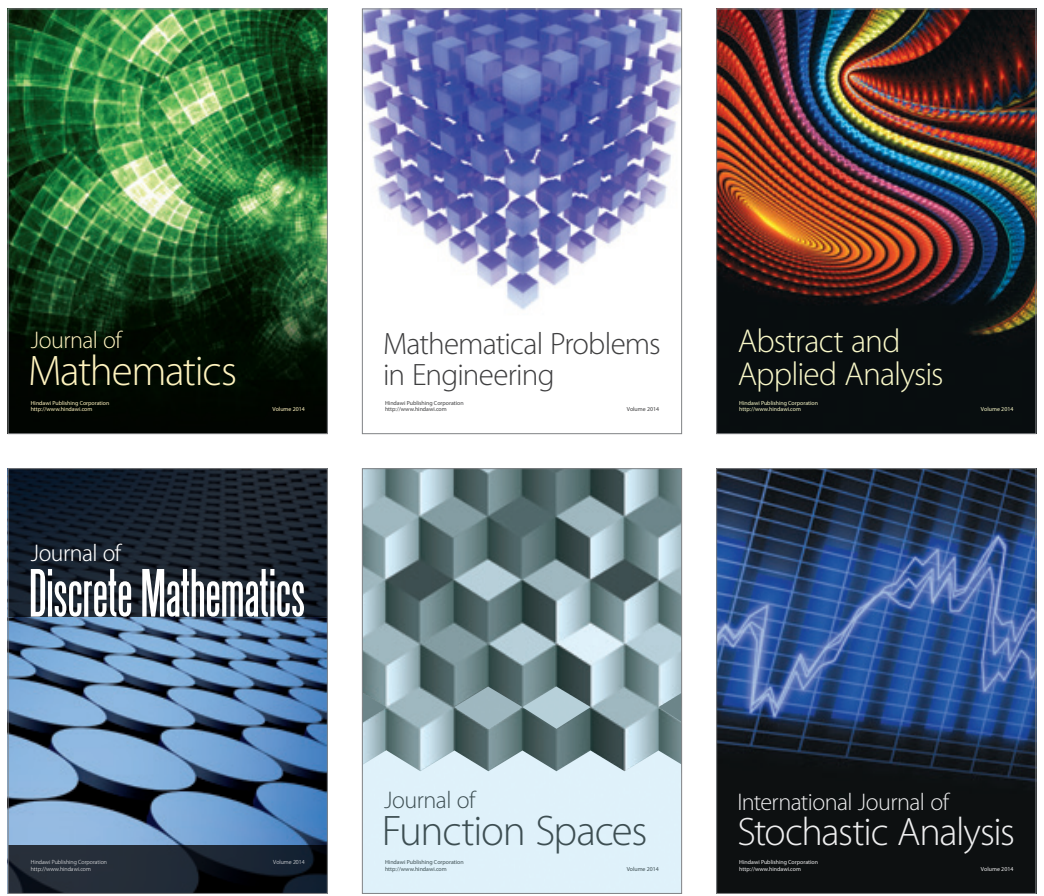

Journal of

Function Spaces

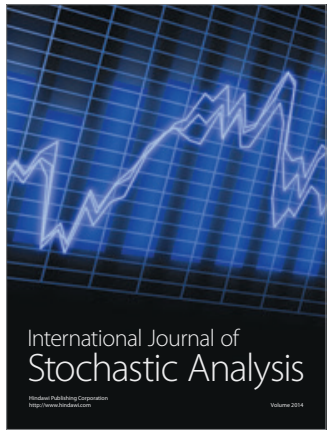

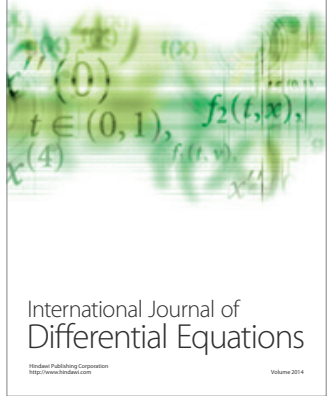
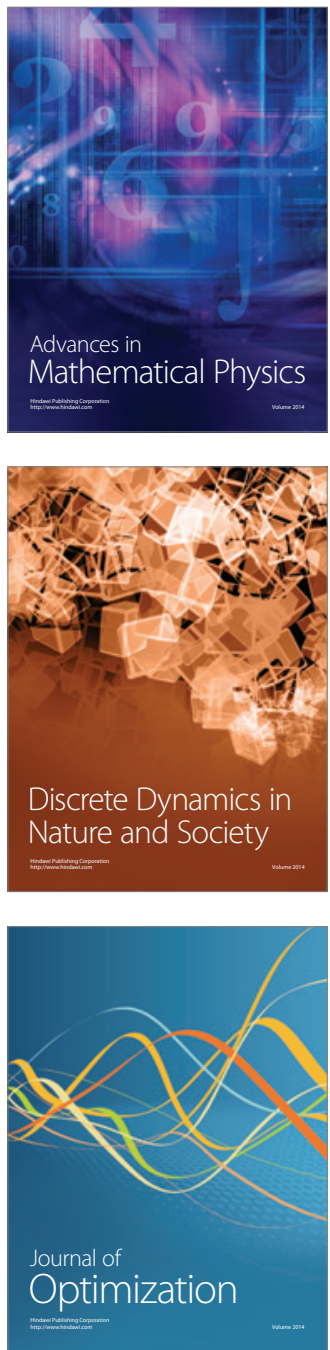